\title{
Validez de la Prueba de Clements en Presencia de Sales Biliares en Líquido Amniótico
}

\author{
Dra. Alcjandra Segovia D. 1; Q.F. M. Eugenia Miller A.2; \\ Dr. Manuel Oyarzún G 1 ; B.Q. Paulina Donoso L. ${ }^{3}$
Effects of Sodium Cholate on Clement's Foam Test in Amniotic Fluid Samples

\begin{abstract}
ln a prcvious study we observed that sodium cholate $\left(5 \times 10^{-5} \mathrm{M}\right.$, average concentration in amriotic fluid from Gravidic Cholestasis) interacts in vitro with pulmonary surfactant. The aim of this study was to determine wether or not sodium cholate $\left(5 \times 10^{-5} \mathrm{M}\right)$ is able to change the result of the foam test developed by Clements et al. Amniotic fluid samples from women without diseases were analized in parallel. The Clements foam test with and witlout sodium cholate (final concentration: $5 \times 10^{-5} \mathrm{M}$ or $10 \times 10^{-5} \mathrm{M}$ ) was performed. No differences were observed.
\end{abstract}

En 1972, Clements y cols. ${ }^{1}$ idearon una prueba semicuantitativa (Prueba de Clements) para determinar la cantidad aproxinada de material tensoactivo, o sea, surfactante pultnonar en liquido amniótico y correlacionar ésta con la maduración pulmonar fetal $y$ el riesgo de desarrollar membrana hialina al nacer.

Buhini $^{2}$, Keniston ${ }^{3}$ y Miller ${ }^{4-5}$ han apreciado la interferencia de sangre $y$ meconio en líquido amniótico sobre los resultados de la prueba de Clements. En un estudio previo ${ }^{6}$, hemos podido observar la interacción in vitro del colato de sodio, a la concentración de $5 \times 10^{-5} \mathrm{M}$, con el surfactante putmonar obtenido de lavados bronquioalveolares de conejos. Estos hallazgos podrian ser interesantes en condiciones tales como la Colestasia Gravidica, patologia muy frecuente en Chile ${ }^{7}$, en que la concentración de ácidos biliares en líquido amniótico está muy elevada (promedio $\left.=5 \times 10^{-5} \mathrm{M}\right)^{8}$, en comparación con embarazos normales: $y$ sugieren la posible interferencia que los ácidos biliares podrían tener sobre las propiedades del líquido amniótico, sobre el surfactante pulmonar fetal y del recién nacido.

1 Médico.

2 Quimico Farmacéutico.

3 Bioupuímico.

Departamento de Medicina Experimental y Obstetricia y Ginecologia, División Ciencias Médicas Oriente, Facultad de Medicina. Universidad de Chile.
El objetivo de este trabajo fue determinar si el colato de sodio a la concentración de $5 \times 10^{--5} \mathrm{M}$ es capaz de modificar la prueba de Clements falseando sus resultados.

\section{MATERIAL Y METODOS}

Se obtuvieron, por lo menos, $8 \mathrm{ml}$ de líquido amniótico por amniocentesis transabdominal de embarazadas sin patologia, que correspondieron a embarazadas con antecedente de cesárea anterior, edad gestacional dudosa o primiparidad tardía. Se usó colato de sodio (99 $100 \%$ pureza Ser. No 90F - 0580, peso molecular 408,6) obtenido de Sigma, St. Louis, Mo. Con $4 \mathrm{ml}$ de cada muestra de líquido amniótico se realizó la prueba de Clements según el método clásico ${ }^{1}$. A los $4 \mathrm{ml}$ restantes se les agregó colato de sodio hasta alcanzar una concentración de $5 \times 10^{-5} \mathrm{M}$ (concentración promedio de ácido cólico en $\mathrm{Co}$. lestasia Gravídica); y luego se realizó la prueba de Clements en forma paralela al control sin colato de sodio. Se analizaron de este modo 10 muestras.

Se realizó además el mismo procedimiento descrito en 7 muestras, alcanzando concentraciones de colato de sodio de $10 \times 10^{-5} \mathrm{M}$.

\section{RESULTADOS}

Con ambas concentraciones de colato de sodio alcanzadas en líquido amniótico no hubo diferencias entre las muestras controles y las que se agregó colato. (Tablas 1 y 2 ). 
Tabla 1

Resultados de la Prueba de Clements en muestras de líquido amniótico control y con colato de sodio $\left(5 \times 10^{-5} \mathrm{M}\right)$

\begin{tabular}{rllc} 
Muestra No & Diagnóstico & \multicolumn{2}{l}{ Prueba de Clements } \\
& & Control & + colato \\
1 & CA & maduro & id. \\
2 & CA & maduro & id. \\
3 & EGD & maduro & id. \\
4 & CA & maduro & id. \\
5 & EGD & maduro & id. \\
6 & EGD & intermedio & id. \\
7 & CA + EGD & intermedio & id. \\
8 & PT & intermedio & id. \\
9 & EGD & intermedio & id. \\
10 & EGD & inmaduro & id.
\end{tabular}

$\mathrm{CA}=$ cesárea an terior

EGD = edad gestacional dudosa

PT = primiparidad tardía

Tabla 2

Resultados de la Prueba de Clements en nuestras de líquido amniótico control y con colato de sodio $\left(10 \times 10^{-5} \mathrm{M}\right)$

\begin{tabular}{lllc} 
Muestra No & Diagnóstico & \multicolumn{2}{l}{ Prucba de Clements } \\
& & Control & + colato \\
1 & CA & maduro & id. \\
2 & CA & maduro & id. \\
3 & EGD & maduro & id. \\
4 & EGD & maduro & id. \\
5 & CA + EGD & intermedio & id. \\
6 & CA & inmaduro & id. \\
7 & CA & inmaduro & id.
\end{tabular}

\section{DISCUSION}

De los métodos para estudiar la madurez pulmonar fetal, a saber: concentración de lecitina, relación lecitina/esfingomielina $(\mathrm{L} / \mathrm{S})$, relación fosfatidilglicerol/fosfatidilinositol ${ }^{9}$, densidad óptica de líquido amniótico a $400 \mathrm{~nm}^{10}$ y prueba de Clements, este último es el más popular en Chile por su simplicidad, accesibilidad y por ser semicuantitativo. La predicción de] riesgo de desarrollar enfermedad de membrana hialina en niños prematuros a través de determinaciones de sutfactante pulmonar en el liquido amniótico ha sido mejorada por la inclusión de la edad gestacional estimada o la determinación de fosfatidilglicerol en la evaluación del niesgo ${ }^{11-12}$. La ausencia de fosfatidilglicerol en el surfactante pulmonar de animales no es incompatible con la vida como ha sido demostrado en experimentos en conejos y en corderos ${ }^{13}$, los cuales presentaban artificialmente bajos niveles de fosfatidilglicerol en surfactante pulmonar sin presentar alteraciones en las propiedades de superficie del surfactante, ni en los gases arteriales y $\mathrm{pH}$. Esos resultados demuestran que a pesar de que el contenido de fosfatidilglicerol en los fosfolípidos pulmonares permite unna excelente predicción de la madurez pulmonar en el feto, su presencia no es esencial para la vida extrauterina pudiendo ser reemplazado por fosfatidilinositol sin causar alteraciones detectables ${ }^{14}$. En Chile la medición de fosfatidilglicerol no es utilizada comúnmente por ser más engorrosa y cara. Además a menudo no se sabe la edad gestacional estimada, ya que una de las causas más frecuentes por las yue se indica la prueba de Clements es el diagnóstico de edad gestacional desconocida y/a dudosa, como puede observarse en nuestro estudio.

Nuestro trabajo demuestra que la prueba de Clements realizada en líquido amniótico de gestaciones aparentemente normales con o sin agregado de colato de sodio en dos concentraciones distintas es igual, o sea, el colato de sodio no modifica la prueba de Clements en las condiciones experimentales de este estudio.

Las observaciones hechas in vitro ${ }^{6}$, en que la adición de colato de sodio hasta alcanzar la concentración de $5 \times 10^{-5} \mathrm{M}$ en lavados bronquioalveolares de conejos, demostró una disminución significativa de las áreas de histéresis de las curvas tensión superficial vs. área, permiten sospechar resultados de madurez pulmonar fetal menores en las muestras con colato con respecto a sus controles. Probablemente esta interacción observada in vitro del colato de sodio a la concentración de $5 \times 10^{-5} \mathrm{M}$ sobre el surfactante pulmonar es neutralizada en la prueba por el etanol utilizado, que actuaría sobre las sales biliares. Por ello seria interesante estudiar la posible interferencia de las sales biliares sobre otras variables de la madurez pulmonar fetal ${ }^{15}$, como por ejemplo, la relación $\mathrm{L} / \mathrm{S}$ y la medición de fosfolípidos totales en líquido amniótico, que guardan gran relación con la prueba de Clements, especialmente cuando esta última es positiva (maduro e intermedio). Otra manera de estudiar el mismo heclo sería correlacionar la madurez pulmonar fetal, determinada por la prueba de Clements, con la edad gestacional en madres con Colestasia Gravídica y gestaciones de edad conocida.

También seria útil correlacionar la prueba de Clements en líquido amniótico de madres con Colestasia del Embarazo y la evolución clínica de los recién nacidos hijos de estas madres, para observar si hay correlación entre Clements negati- 
vo y sindrome de Dificultad Respiratoria Idiopático o si existen falsos positivos con prueba de Clements maduro y recién nacidos con esta patología respiratoria.

Cabe además preguntarse qué ocurre con el surfactante fetal y del recién nacido, en que supuestamente no habría sustancias neutralizadoras de las sales biliares, como el etanol, ni otros procedimientos de laboratorio que puedan interferir en la acción de tales sales sobre el surfactante pulmonar.

\section{RESUMEN}

En un estudio previo observamos que el colato de sodio $\left(5 \times 10^{-5} \mathrm{M}\right.$, concentración promedio en líquido amniótico en Colestasia Gravídica) interfiere in vitro con el surfactante pulmo. nar. El objetivo de este trabajo fue determinar si el colato de sodio $\left(5 \times 10^{-5} \mathrm{M}\right)$ es capaz de falsear los resultados de la prueba de Clements. En muestras de liquido amniótico de embarazadas sin patología se realizó en forma paralela la prueba de Clements sin y con colato de sodio, agregado hasta alcanzar concentraciones de $5 \mathrm{x}$ $10^{-5} \mathrm{M}$ o $10^{-4} \mathrm{M}$. En este trabajo los resultados de la prueba no fueron falseados por la adición de colato de sodio hasta alcanzar las concentraciones mencionadas. Sin embargo, este hecho no nos permite afirmar que el surfactante pulmonar fetal y del recién nacido no sean afectados in vivo por las sales biliares, condiciones en que éstas no serían neutralizadas por el etanol como ocurre en la prueba.

\section{REFERENCIAS}

1 Clements, J.. Platzker, A., Tiemey, D., Hobel, C., Creasy, R., Margolis, A., Thibeault, D., Tooley. W. and $O h, W_{*}$ Assessment of the risk of the respiratory-distress syndrome by a rapid test for surfactant in amniotic fluid. N. Engl. J. Med. 286: 1077, 1972.
${ }^{2}$ Buhi, W.: Effects of blood or meconium on the determination of the amniotic fluid Iccitin/sphingo. myyetin ratio. Am. J. Obstet, Gynecol. 121: 321, 1975.

${ }^{3}$ Keniston, $R$. et al.: The effect of blood, meconium and temperature on the rapid surfactant test. Obs. tet. Gynecol. 48: 442, 1976.

4 Miller, $M$., Tapia, J.: Efecto de la sangre y meconio sobre el Test de Clements. (Comunicación personal), L981.

${ }^{5}$ Miller. M.: Importancia de los parámetros bioqujmicos del líquido amniótico en el diagnóstico prenatal. Informe final I Congreso de Quínica Clinica. C-9 pp 1-13, Santiago, 1980.

- Donoso, P., Oyarzun, M., Segovia, A., Guerrero, $M$. and Puig, $F .:$ Sodium cholate interactions with rabbit's pulmonary surfactant. Biol. Neonate. (En prensa).

7 Avendafio, O.: Esquemas de Obstetricia. Patología del Embarazo. Santiago. Ed. Universitaria, 1979.

${ }^{8}$ Heikkimen, 1., Mäentansta, R., Tuimala, R., YlöstaLo. $P$. and Jönna, $O$.: Amniotic fluid bile acids in nomal and pathological pregnancy. Obstet. Gynec. 56: $60,1980$.

${ }^{9}$ Hollman, M. Kulovich, M. Kirkpatrick, E., Sugarman, $G$. and Gluek, L.: Phosphatiaylinositol and phosphatidulglycerol in amniotic fluid: indices of lung maturity. Am. J. Obstet. Gynecol. 125: 613, 1976.

10 Sbarra, J., Michlewitz, $H$, et cols.: Correlation between amniotic fluid optical density and $L / S$ ratio. Obstet. Gynecol. 48: 613, 1976.

1 I Schlueter, M., Phibbs, R., Creasy, R., Clements, J. and Tooley, W.: Antenatal prediction of graduated risk of hyaline membrane disease by amsiotic fluid foam test for surfactant. Am. J. Obstet. Gynecal. 134: 761, 1979.

12 Clements, J.: Ontogenia y métodos de detección del surfactante pulmonar. Rev. Méd. Chile. 110: 819, 1982.

13 Benson, B., Kitterman, J., Mescher, E. and Tooley, $W$.: Changes in phospholipid composition of lung surfactant during development in the fetal lamb. Biochimica et Biophysica Acta. 753: 83, 1983.

14 Beppu, $O$., Clements, $J$, and Goerke, $J$.:Phophatidylglycerol deficient lung surfactant has normal properties, 3. Appl. Phy siol.: Respirat. Environ. Exercise Physiol. 55: 496, 1983.

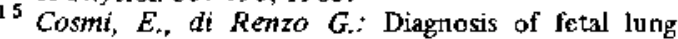
maturity, in Pulmonary Surfactant System. E. Cosmi y E. Scarpelli eds. Elsevier Science Publishers, Anstèdam, 1983 pp: $75-97$. 